\title{
Inelastic Scattering of CN Radicals at the Gas-Liquid Interface Probed by Frequency-Modulated Absorption Spectroscopy
}

\author{
Paul D. Lane, Katya E. Moncrieff, Stuart J. Greaves, Kenneth \\ G. MCKendrick and Matthew L. Costen*.
}

Institute of Chemical Sciences, School of Engineering and Physical Sciences, Heriot-Watt University, Edinburgh, EH14 4AS, United Kingdom

\section{Supporting Information}

4 Pages, 2 Figures, 0 Tables 


\section{S1: Incident molecular beam Doppler profiles and transverse speed distributions as a function}

of discharge-probe delay

The appearance profile for the $j=0.5$ state in the incident molecular beam, shown in Figure 2(a) in the paper, displays a clear shoulder at later times $(\approx 170-190 \mu \mathrm{s})$. Doppler lineshapes for different discharge-probe delays, and the transverse speed distributions arising from fitting to those lineshapes, demonstrate that the structure in the appearance profile is associated with changes in the transverse speeds. Figure S1(a) shows the experimental Doppler lineshapes at 4 different delays, $156 \mu \mathrm{s}, 164 \mu \mathrm{s}$, $176 \mu$ s and $192 \mu \mathrm{s}$. These cover the peak of the incident beam, the $50 \%$ fall point, the start of the shoulder, and the end of the shoulder, respectively. Clear changes are visible in the both the lineshapes and the fitted speed distributions as a function of delay. At the peak of the appearance profile the transverse speed distribution is narrow and essentially symmetrically peaked around $\approx$ $500 \mathrm{~ms}^{-1}$. We interpret this as a well-defined packet of $\mathrm{CN}$ embedded in the majority He free-jet expansion, with the transverse speed distribution defining the cone angle of the free-jet. The peak of the transverse speed distribution initially moves to higher speeds as the delay increases to $164 \mu \mathrm{s}$, consistent with this later delay preferentially probing parts of the $\mathrm{CN}$ packet that have taken more offaxis paths. The speed distribution then undergoes a substantial change at the shoulder of the appearance profile, $176 \mu \mathrm{s}$. The peak transverse speed reduces to $\approx 500 \mathrm{~ms}^{-1}$ again, but the spread of speeds increases substantially. Finally, at the last delay, $192 \mu$ s, the speed distribution has narrowed again, with the peak speed increasing to $\approx 700 \mathrm{~ms}^{-1}$. This last delay corresponds to an on-axis speed of $1540 \mathrm{~ms}^{-1}$. We do not have a clear explanation for this detailed late-time structure, but believe it represents a combination of off-axis boundary shockwaves in the free-jet expansion, and the dynamics of the pulsed discharge and the cooling and entrainment of the $\mathrm{CN}$ generated therein. The relative contributions of these factors could perhaps be explored in future work through skimming of the molecular beam to remove the boundary shockwaves. 

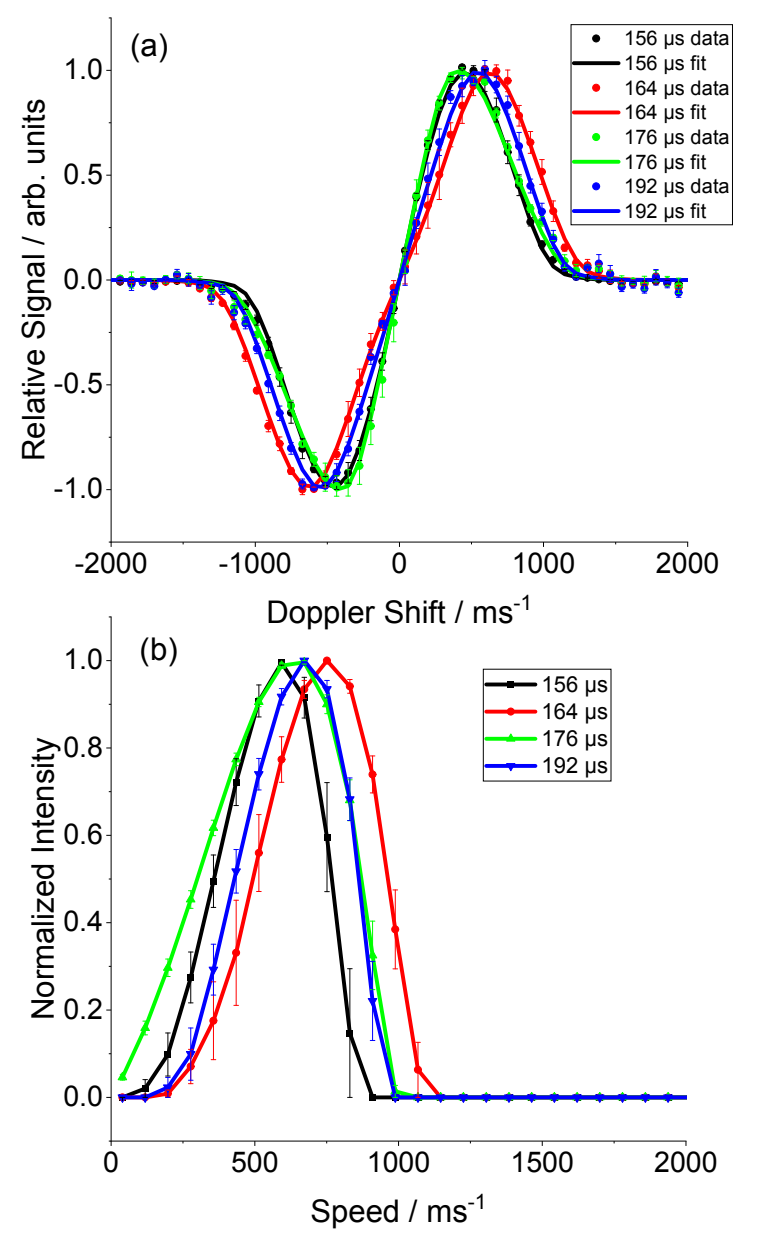

Figure S1: (a) Absorption Doppler lineshapes for the incident molecular beam in $j=0.5$, at 4 different discharge-probe delays. Symbols are experimental data, and solid lines are fits to extract the speed distribution. $156 \mu$ s (black), $164 \mu$ s (red), $176 \mu$ s (green) and $192 \mu$ s (blue). (b) Transverse speed distributions from the fits to lineshapes in (a).

\section{S2: Doppler profiles for scattered product states}

Figure S2 shows Doppler lineshapes and fits as a function of appearance time (rising edge, peak and falling edge) for 3 different rotational states, $j=6.5,12.5$ and 18.5 , together with a simulated lineshape for a $298 \mathrm{~K}$ Maxwell-Boltzmann speed distribution. The data and fits in S2(a) are also shown in figure 6 of the main paper, and here we provide two higher rotational states for comparison. In all cases, the experimental lineshapes are substantially wider than the reference Maxwell- 
Boltzmann, implying that impulsive scattering dominates the speed distribution for all rotational levels and at all times. There is also a clear variation in Doppler lineshape with appearance time, with a consistent order in width: rising edge $>$ peak $>$ falling edge. As discussed in the main paper, this is consistent with the speeds normal and parallel to the surface being strongly correlated, consistent with impulsive scattering with a broad angular distribution.
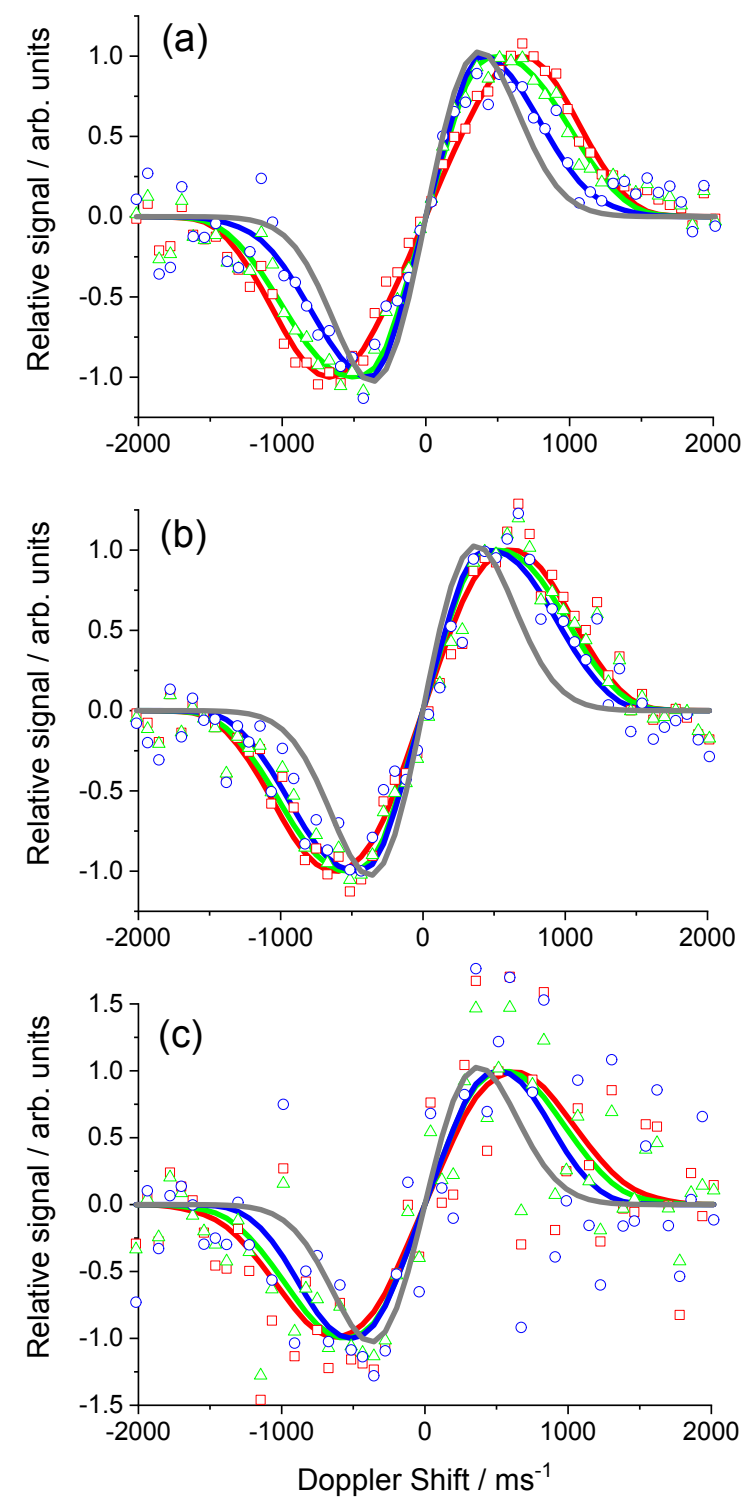

Figure S2: Doppler profiles of scattered signal in (a) $j=6.5$, (b) $j=12.5$, (c) $j=18.5$ data for rising edge (squares), peak (triangles), falling edge (circles) and fits to the rising edge (red line), peak (green line), falling edge (blue line), compared to a thermal distribution at $298 \mathrm{~K}$ (grey line). 